\title{
Effect of lipopolysaccharide derived from surabaya isolates of Actinobacillus actinomycetemcomitans on alveolar bone destruction
}

\author{
Rini Devijanti Ridwan ${ }^{1}$, Sidarningsih ${ }^{1}$, Tuti Kusumaningsih ${ }^{1}$ and Sherman Salim²
}

1. Department of Oral Biology, Faculty of Dental Medicine, Universitas Airlangga, Surabaya, Indonesia; 2. Department of Prosthodontic, Faculty of Dental Medicine, Universitas Airlangga, Surabaya, Indonesia.

Corresponding author: Rini Devijanti Ridwan, e-mail: rini-d-r@fkg.unair.ac.id

Co-authors: Sidarningsih: sidarningsih@fkg.unair.ac.id, TK: tuti-k@fkg.unair.ac.id, SS: sherman-s@fkg.unair.ac.id Received: 11-10-2017, Accepted: 03-01-2018, Published online: 10-02-2018

doi: 10.14202/vetworld.2018.161-166 How to cite this article: Ridwan RD, Sidarningsih, Kusumaningsih T, Salim S (2018) Effect of lipopolysaccharide derived from surabaya isolates of Actinobacillus actinomycetemcomitans on alveolar bone destruction, Veterinary World, 11(2): 161-166.

\begin{abstract}
Background: Actinobacillus actinomycetemcomitans' lipopolysaccharide (LPS) has a high virulence factor. It interacts with serum protein through receptors on the epithelial cell surface, thereby increasing both interleukin (IL)-1 $\beta$, and IL-6 which results in damage to periodontal tissue.
\end{abstract}

Aim: The aim of the study was to identify and evaluate the effect of LPS derived from local isolates (A. actinomycetemcomitans)
on the destruction of alveolar bone by means of several biomarkers, including; the number of osteoblasts and osteoclasts, the
expression of IL-6, matrix metallopeptidase 1 (MMP-1), and receptor activator of nuclear factor kappa-B ligand (RANKL).

Materials and Methods: The isolation of LPS from A. actinomycetemcomitans was calculated using phenol, while purification was performed using Sephadex C-18 column chromatography. 40 Wistar rats were divided into four groups of 10. Each treatment was divided into two groups which were $0.9 \% \mathrm{NaCl}$ and LPS induced for 7 and 14 days, respectively. Gingival and alveolar bones were further introduced into the induction area, followed by the measuring of osteoblast and osteoclast with hematoxylin-eosin staining, IL-6, MMP-1 and RANKL expression with immunohistochemical.

Results: Reduced numbers of osteoblasts at the $7^{\text {th }}$ and $14^{\text {th }}$ day of treatment were detected, while those of osteoclasts increased. There was an increased expression of IL-6, MMP-1, and RANKL in the $7^{\text {th }}$ and $14^{\text {th }}$-day treatment group. Treatment of LPS from A. actinomycetemcomitans over 7 and 14 days resulted in damage to periodontal tissue and alveolar bone in Wistar rats.

Conclusion: LPS of A. actinomycetemcomitans administration for 7 and 14 days causes periodontal and alveolar tissue destruction in Wistar rats.

Keywords: Actinobacillus actinomycetemcomitans local isolate, lipopolysaccharide, interleukineIL-6, matrix metallopeptidase-1, receptor activator of nuclear factor kappa-B, receptor activator of nuclear factor kappa-B ligand.

\section{Introduction}

Lipopolysaccharide (LPS) is a component of A. actinomycetemcomitans forming its cell wall as a virulence factor. LPS interacts with serum protein through receptors on the epithelial cell surface [1]. The high concentration of LPS increases interleukin (IL) $-1 \beta$ and IL- 6 which, in turn, results in the destruction of periodontal tissue due to a cytokine response in the epithelium, neutrophil, fibroblast, and monocyte. The process occurs through host stimulation and plays an important role in tissue damage by stimulating the macrophage to release (IL-1), IL-1 $\beta$, and tumor necrosis factor (TNF). These pro-inflammatory cytokines are responsible for causing bone damage [2].

The LPS interferes with the homeostasis of collagen metabolism through collagen phagocytosis through

Copyright: Ridwan, et al. Open Access. This article is distributed under the terms of the Creative Commons Attribution 4.0 International License (http://creativecommons.org/licenses/ by/4.0/), which permits unrestricted use, distribution, and reproduction in any medium, provided you give appropriate credit to the original author(s) and the source, provide a link to the Creative Commons license, and indicate if changes were made. The Creative Commons Public Domain Dedication waiver (http:// creativecommons.org/publicdomain/zero/1.0/) applies to the data made available in this article, unless otherwise stated. fibroblast. A study by Shaddox et al. [3], found that LPS stimulates the synthesis of osteoblastic IL- $1 \beta, \mathrm{TNF} \alpha$, IL-6, and receptor NF-KB ligand (RANKL). The virulence potential of the A. actinomycetemcomitans might be different. Specific Polysaccharide Antigen (SPA), serotype $\mathrm{b}$ of $A$. actinomycetemcomitans is responsible for the resistance mechanism to phagocytosis and kills polimorfonuclear leukocyte in humans [4]. Takahashi et al. [4] reported that the SPA of serotype $\mathrm{a}$ and $\mathrm{c}$ induces IL-1 release by murine's macrophage which is lower than the SPA of A. actinomycetemcomitans serotype b. Meanwhile, Patil et al. [5] demonstrated the higher sensitivity of various antimicrobials in other serotypes compared to that of A. actinomycetemcomitans. Some kinds of yeasts secrete toxins and these types called killer yeasts. These yeasts can inhibit the growth of other yeast strains and also have antimicrobial activities inhibiting growth of bacteria $[6,7]$.

The aims of this study were to determine the effect of LPS in a local isolate of A. actinomycetemcomitans, as characterized by several markers of damaged alveolar, through the measuring of osteoblast and osteoclast, as well as IL-6, Matrix Metallopeptidase 1 (MMP-1) and RANKL expression. 


\section{Materials and Methods}

\section{Ethical approval}

This study received an ethical clearance approval letter relating to human subjects from the Ethics Research Committee of Faculty of Dental Medicine, Universitas Airlangga, with number 29/KKEPK/ FKG/III/2015. The research constituted Analytical Observational involving the use of an Experimental Laboratory and a cross-sectional method.

\section{A. actinomycetemcomitans local isolate preparation}

A. actinomycetemcomitans was obtained from patients with aggressive periodontitis and further subcultured in Luria Berthani (Merck KgaA ${ }^{\circledR}$, Darmstadt, Germany) for 2-3 days to obtain its morphology (visualized in 2 plates). An $A$. actinomycetemcomitans culture of $200 \mu \mathrm{l}$ was required for four animal model groups (40 rats in total); therefore, $10 \mathrm{ml}$ culture was stored at a density of $10^{8}$ cells every day for a period of 14 days. $200 \mu \mathrm{l}$ LPS of $A$. actinomycetemcomitans was produced containing $200 \mu \mathrm{g} / \mathrm{ml}$ proteins to induce sulcus of the maxilla molar of Wistar rats over $7^{\text {th }}$ day to $14^{\text {th }}$ day. LPS was isolated and purified following Westphal and Jann's [6] phenolic-based method. The resulting purified crude LPS and isolate were subsequently gel-filtrated in Sephadex ${ }^{\circledR}$ C-18 (Sigma

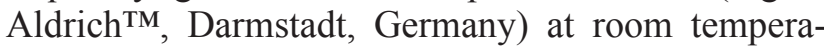
ture with a disaggregation buffer as solvent $(0.05 \mathrm{M}$ Tris-HCl pH 9, 0.001 M EDTA, and 0.3 deoxycholate) (Sigma-Aldrich $^{\mathrm{TM}}$, Darmstadt, Germany).

\section{Animal model study preparation}

The animal model used consisted of Wistar rats divided into four groups each containing 10 rodents. Group 1 constituted a control group, induced with $\mathrm{NaCl} 0.9 \%$ for 7 days Group 2 received treatment with LPS for 7 days. Group 3 was induced with $\mathrm{NaCl} 0.9 \%$ for 14 days, while Group 4 underwent treatment with LPS for 14 days [4,5].

Treatment was injected into the sulcus of the first maxillary molar (M1) following Dumistrescu's method [8,9]. Each group was divided into two subgroups, one treated for 7 days, the other for 14 days. Injections of $200 \mu \mathrm{g}$ of LPS, with a protein level of $200 \mu \mathrm{g} / \mathrm{ml}$, and a density of 108 were administered for at least 7 days to obtain significant aggressive periodontitis symptoms [9]. Gingival and alveolar bones were subsequently examined into the induction area, followed by the measuring of osteoblast and osteoclast with hematoxylin-eosin (HE) staining, and IL-6, MMP-1, and RANKL expression with an immunohistochemical marker kit (Merck KgaA ${ }^{\circledR}$, Darmstadt, Germany).

At the next immunohistochemical examination on glass object with $50 \%$ glycerin dropped above the glass object and viewed the results with an Electron Microscope (Automated Electron Microscope, Olympus $^{\circledR}$, USA). Data in normal distribution were analyzed using Shapiro-Wilk test $(\mathrm{p}>0.05)$.

\section{Statistical analysis}

Data homogeneity was analyzed using ANOVA $(\mathrm{p}<0.05)$. Statistical analysis was effected by means of Statistical Package for the Social Sciences (SPSS) 17.0 software for windows 8.1 by SPSS Inc., Chicago, United States.

\section{Results}

\section{Measurement of osteoblast and osteoclast}

The measurement of osteoblast from alveolar bones on day 7 showed data were normally distributed and homogenous with a significant value of $\mathrm{p}>0.05$. ANOVA analysis showed there was a considerable difference on day 7 in the osteoblast between the treatment group $16.5 \pm 3.84$ and the control group $5 \pm 0.82$ $(\mathrm{p}<0.05)$. Measurement of osteoblast from alveolar bones carried out on day 14 showed was normally distributed and homogenous $p>0.05$. ANOVA analysis showed there was a marked difference in osteoblast between the treatment group 22.6 2 2.41 on day 14 and that of the control group $5.8 \pm 1.48(\mathrm{p}<0.05)$.

The measurement of osteoclast from alveolar bones on day 7 showed data to be normally distributed and homogenous $\mathrm{p}<0.05$. ANOVA analysis showed there was a no stable contrast in osteoclast between the treatment group on day 7 and the control group $(\mathrm{p}<0.05)$. The measurement of osteoclast from alveolar bones on day 14 confirmed that data were normally distributed and homogenous with a significant value of $p>0.05$. ANOVA analysis showed there was a considerable difference in osteoclast between the treatment group on day 14 and the control group $(\mathrm{p}<0.05)$.

A graphic of the average alveolar osteoblast on day 7 showed 15 cells in the control group and 7.4 cells in the treatment group. The measurement of osteoclast from gingiva tissue on day 7 confirmed there to be 5 cells in the control group and 16.5 cells in the treatment group. Osteoblast measurements taken from the alveolar on day 14 showed that osteoblast represented 22.6 cells in the control group, but only 4.5 cells in the treatment group. The measurement of osteoclast-derived from gingiva tissue on day 14 established the presence of 5.8 cells in the control group, in contrast to the 22.6 cells in the treatment group. The average presence of osteoblast and osteoclast in the alveolar bone can be seen in Figure-1, while the results of $\mathrm{HE}$ staining of gingiva tissue are shown in Figure-2.

\section{Expression of IL-6, MMP-1, and RANKL}

The expression of IL- 6 in gingival tissue during day 7 showed data were normally distributed and homogenous $(\mathrm{p}>0.05)$. ANOVA analysis revealed a significant difference of IL-6 expression between the day 7 treatment group $15.8 \pm 0.79$ and the control group $4.7 \pm 1.16(\mathrm{p}<0.05)$. The expression of IL-6 from gingival tissue undergoing day 14 treatment showed the data to be normally distributed and homogenous $(p>0.05)$. ANOVA analysis negated any significant difference of IL-6 expression between 
the day 14 treatment group $2.1 \pm 0.74$ and the control group $1.9 \pm 0.74(p<0.05)$. The average IL-6 expression in gingival tissue is shown in Figure-3, while the result of IHC staining of Wistar rat gingiva features in Figure-4.

The data of MMP-1 expression in gingiva in day 7 treatment were normally distributed and homogenous $(p>0.05)$. ANOVA analysis confirmed a considerable difference in IL- 6 expression between the day 7 treatment $15.3 \pm 0.67$ and control groups $6.1 \pm 0.99(p<0.05)$. The data of MMP-1 expression in gingiva during day 14 treatment were normally distributed and homogenous $(p>0.05)$. ANOVA analysis confirmed a significant difference in IL-6 expression between the day 14 treatment group $5.9 \pm 0.74$ and the control group $3.9 \pm 0.74$ $(\mathrm{p}<0.05)$. The average MMP-1 expression in gingival tissue is shown in Figure-5, with the result of IHC staining of Wistar rat gingiva contained in Figure-6.

The data of RANKL expression in gingiva after treatment lasting 7 days was normally distributed and homogenous ( $p>0.05)$. ANOVA analysis showed there was a significant difference in RANKL expression between the day 7 treatment group $15 \pm 2.5$ and the control group $10.1 \pm 2.5(\mathrm{p}<0.05)$. Data relating to RANKL

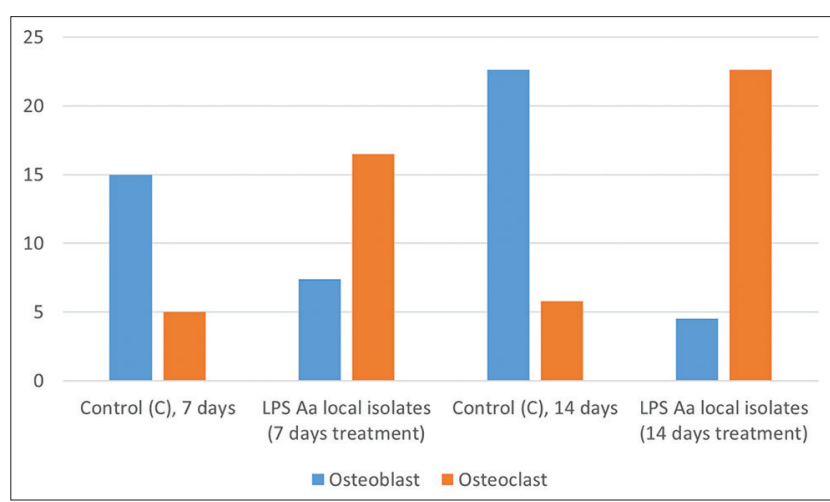

Figure-1: Average levels of osteoblast and osteoclast in treatment on days 7 and 14 in alveolar bone.

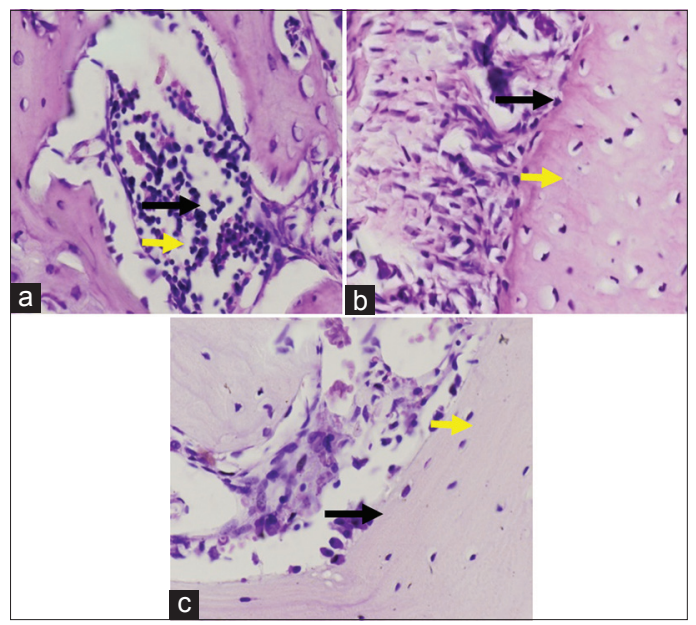

Figure-2: Hematoxilin-Eosin staining of gingiva tissue. Control (a); with 7-day induction of lipopolysaccharide (LPS) (b); Group with 7-day induction of LPS (c). The black arrow indicates osteoclast, while the yellow shows the location of osteoblast. expression in gingiva after 14-day treatment was normally distributed and homogenous ( $p>0.05$ ). ANOVA analysis showed there to be a significant difference in RANKL expression between the day 14 treatment group $18.8 \pm 1.03$ and the control group $15 \pm 1.2$ $(p<0.05)$. The average RANKL expression in gingiva is shown in Figure-7 with the result of IHC staining of Wistar rat gingiva contained in Figure-8.

\section{Discussion}

The highest concentration of osteoblasts in the alveolar bone was found in the day 14 control group and differed significantly compared to that of the treatment group. These results occurred due to the absence of aggressive periodontitis which, in contrast, was found to be present in the LPS treatment group. Osteoblast plays an important role in mineralization and hydroxyapatite precipitation by regulating calcium and phosphate levels during the composition of hydroxyapatite. Osteoblast produces alkaline phosphatase in adequate amounts in the plasma membrane responsible for bone mineralization. Osteoblast also produces cytokines such as colony stimulating

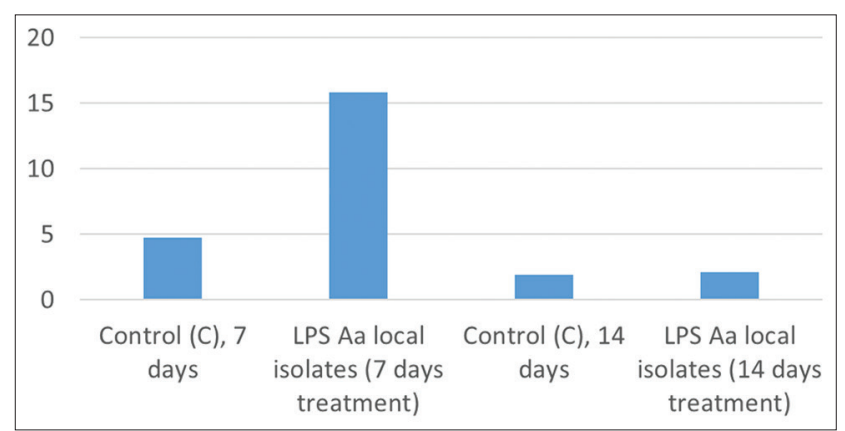

Figure-3. Average interleukin-6 expression in gingival tissue.

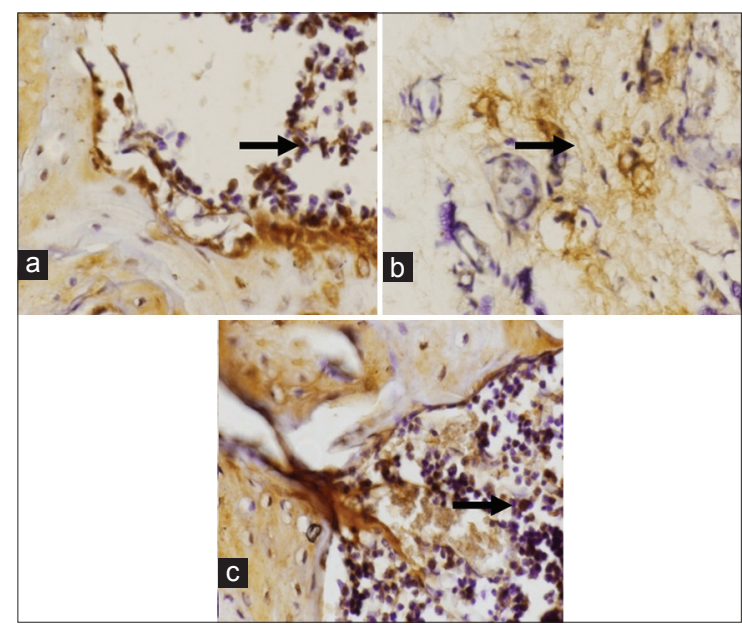

Figure-4: Expression of interleukin-6 in gingiva of Wistar rat. Control (a); Actinobacillus actinomycetemcomitans lipopolysaccharide (LPS) (day 7) (b); A. actinomy cetemcomitans LPS (day 14) (c), indicated by the brown regions in the nucleus (black arrow). Activated expression of interleukin - 6 was shown as brown in cytoplasm of gingiva epithelium (black arrow) at $400 \times$ magnification. 


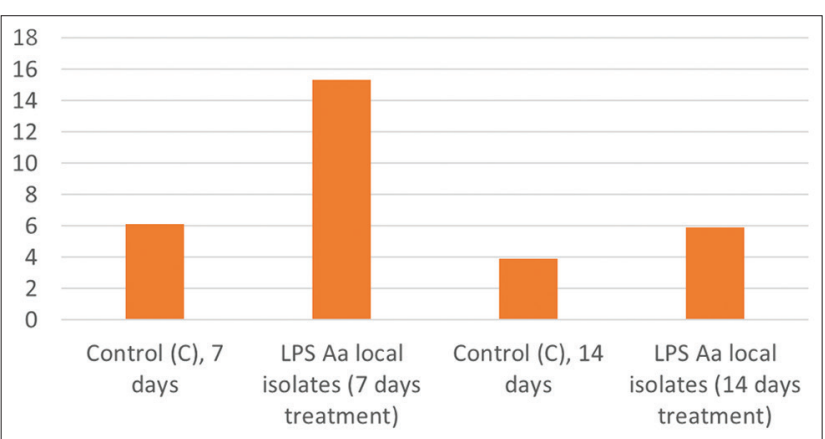

Figure-5. Average matrix metallopeptidase-1 expression in gingiva.

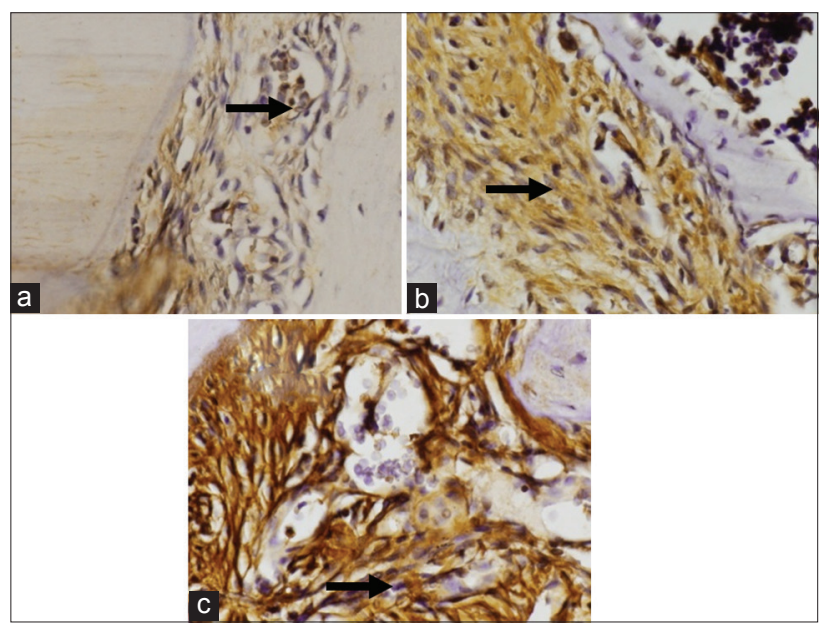

Figure-6: Matrix metallopeptidase-1 (MMP-1) expression in gingiva of Wistar rat. Control (a); treatment of lipopolysaccharide (LPS) (7-day) (b); treatment of LPS (14-day) (c), shown as brown in the nucleus (black arrow). Activated MMP-1 expression is highlighted as brown in cytoplasm of gingiva epithelium (black arrow) at $400 \times$ magnification.

factor-1, RANKL, and osteoprotegerin [8-10]. The highest concentration of alveolar osteoclast was found in the day 14 LPS treatment group and differed considerably compared to that of the control group.

The induction of LPS causes adhesion and invasion in the host. LPS is a major factor in bacteria and plays an important role in bone resorption through osteoclast stimulation in which LPS activates osteoblast to release factors attracting and activating osteoclast [11]. LPS can make macrophages activated, and can also trigger the synthesis o-f both cytokines that have pro-inflammatory activities, such as interleukin (IL)-1, IL-6, IL-8, and TNF $\alpha$, as well as another cytokine, namely, IL-10 serving as regulator $[2,3,12]$. LPS also inhibits collagen and non-collagen protein synthesis. Referring to a study conducted by Takahashi et al [4] and Nair et al. [13] found that the LPS of A. actinomycetemcomitans causes bone resorption in murine calvarial by stimulating murine macrophage. A. actinomycetemcomitans causes alveolar bone destruction and antibody response in three rat strains; Fawn Hooded Hypersensitive, Dahl Salt Sensitive, and Norway Brown [14].

The process of periodontitis passes through 4 phases: (1) Accumulation and presence of bacteria

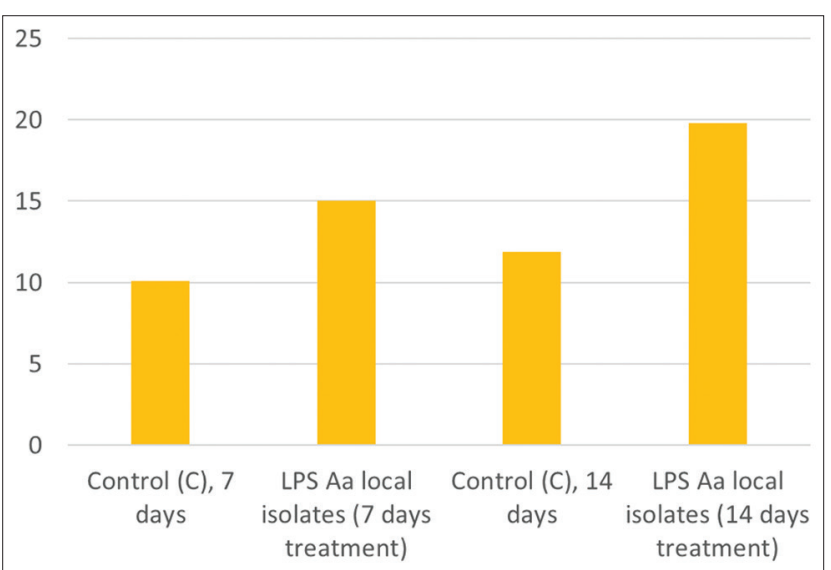

Figure-7: Average receptor activator of nuclear factor kappa-B ligand expression in alveolar.

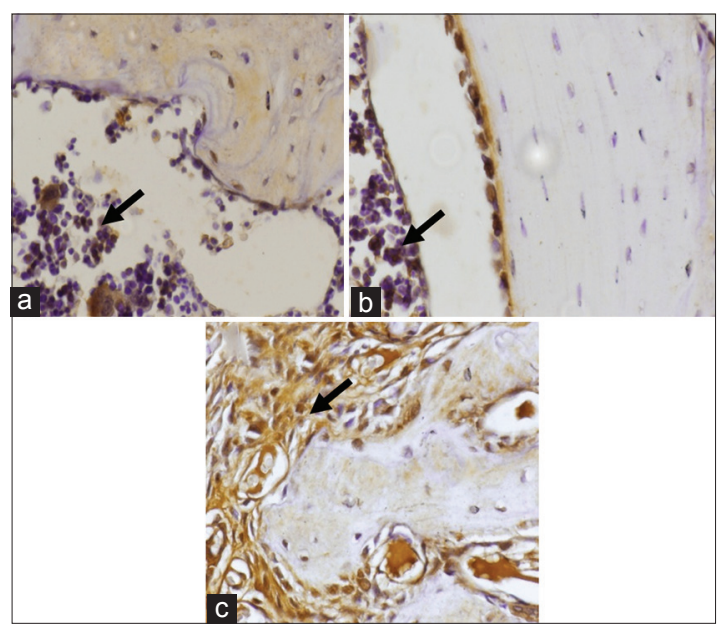

Figure-8: Expression of receptor activator of nuclear factor kappa-B ligand in alveolar of Wistar rat. Control (a); Actinobacillus actinomycetemcomitans lipopolysaccharide (7 days) (b); A. actinomycetemcomitans (14 days) (c), shown as brown in the nucleus (black arrow) at a magnification of $400 \times$.

in gingival sulcus (colonization); (2) bacterial invasion of the epithelium and gingiva; (3) host response stimulation, acquired and innate (inflammation) immune response activation; and (4) destruction of connective tissue attachment on dental enamel and bone causing irreversible damage [1]. The presence of LPS decreases osteoblast. LPS generates stimulation on RANKL which further binds to RANK, stimulating tumor necrosis receptor-associated factors- 6 for osteoclast progenitor activation leading to osteoclast differentiation, activation and an increased number of osteoclasts. This, in turn, causes alveolar bone destruction which can serve as a marker if such destruction has occurred [15].

IL-6 expression in gingiva during day 7 treatment showed an increase and a marked difference compared to the control, indicating the presence of LPS in periodontal tissue. LPS stimulates pro-inflammatory cytokine activation, such as IL-6, stimulating bone resorption, and inhibiting bone formation leading to periodontal and alveolar damage $[2,16]$. 
The expression of MMP-1 in the day 7 and day 14 treatment groups showed a significant contrast compared to that of the control group. The induction of LPS A. actinomycetemcomitans causes pro-inflammatory cytokine stimulation. IL-8 produced by monocyte, keratinocyte, endothelial, and fibroblast cell stimulates the release of MMPs by neutrophil. One such MMP was MMP-1 which is a potential collagenase- 1 and plays a role in the degradation of connective tissue in the inflamed area. The high expression of MMP-1 provokes periodontal and alveolar damage in accordance with the study conducted by Claesson et al. [17] that confirmed A. actinomycetemcomitans to be a strong stimulant to MMP-8 release, mainly due to Leukothera being one of its 15 virulence factors.

The expression of RANKL in alveolar bones tends to increase and shows a significant difference in the day 7 and day 14 treatment groups compared to the control group. The result confirms LPS-1 as an antigenic component raising host immune response and further stimulating pro-inflammatory cytokines, especially TNF- $\alpha$ and IL-1. TNF- $\alpha$ and IL-1 stimulate RANKL which increase osteoclast. The high expression of osteoclast augments osteocalcin that can lead to alveolar bone resorption. A previous study conducted by Bullon et al. [18], posited that the increased number of osteocalcins was a marker of bone formation inhibition. An elevation osteocalcin level in serum was associated with the rate of bone destruction. Studies involving animals confirmed the role of osteocalcin in alveolar bone resorption.

\section{Conclusion}

LPS induces IL-6 expression, osteoclastogenesis, and bone resorption. Injection of porphyromonas gingivalis from IL-6-removed rats causes a reduced level of bone loss. This implies that IL-6 contributes to bacteria progressivity which promotes alveolar damage. RANKL regulates the pathological and physiological conditions of bone resorption. During the pathological inflammation phase of the bone disease, RANKL expression was found to be present in B cell, $\mathrm{T}$ cell, and monocyte. Activation of T-cell and B-cells are cellular sources of RANKL in bone resorption in gingiva during inflammation. These results support the conclusion that LPS of A. actinomycetemcomitans administration over day 7 and day 14 causes periodontal and alveolar damage in Wistar rats.

\section{Authors' Contributions}

Conception and design of the study: RDR, TK Acquisition of data:S RDR, Sidarningsih, Analysis and/or interpretation of the data: RDR, SS. Drafting and revising the manuscript critically for important intellectual content: RDR, TK and SS. All authors read and approved the final manuscript.

\section{Acknowledgments}

The authors would like to thank the Faculty of Dental Medicine. Research grant is funded by
Directorate of Research and Community Service, Directorate General of Strengthening Research and Development of Research, Technology and Higher Education Ministry: Letter of Appointment Agreement of Research Program number: 018/SP2H/LT/DRPM/ II/2016, February 17, 2016

\section{Competing Interests}

The authors declare that they have no competing interests.

\section{References}

1. Luo, Q., Yang, X., Yu, S., Shi, H., Wang, K., Xiao, L., Zhu, G., Sun, C., Li, T., Li, D. and Zhang, X. (2017) Structural basis for lipopolysaccharide extraction by ABC transporter LptB2FG. Nat. Struct. Mol. Biol., 24: 469-474.

2. Tessaro, F.H., Ayala, T.S., Nolasco, E.L., Bella, L.M. and Martins, J.O. (2017) Insulin influences LPS-induced TNF- $\alpha$ and IL-6 release through distinct pathways in mouse macrophages from different compartments. Cell Physiol. Biochem., 42: 2093-2104.

3. Shaddox, L.M., Gonçalves, P.F., Vovk, A., Allin, N., Huang, H., Hou, W., Aukhil I. and Wallet, S.M. (2013) LPSinduced inflammatory response after therapy of aggressive periodontitis. J. Dent. Res., 92: 702-708.

4. Takahashi, N., Kobayashi, M., Takaki, T., Takano, K., Miyata, M., Okamatsu, Y., Hasegawa, K., Nishihara, T. and Yamamoto, M. (2008) Actinobacillus actinomycetemcomitans lipopolysacharide stimulates collagen pagocytosis by human gingival fibroblast. Oral Microbiol. Immunol., 23: 259-264.

5. Patil, C., Rossa, C. Jr. and Kirkwood, K.L. (2006) Actinobacillus actinomycetemcomitans lipopolysaccharide induces interleukin-6 expression through multiple mitogen-activated protein kinase pathways in periodontal ligament fibroblasts. Oral Microbiol. Immunol., 21: 392-398.

6. Janus, M.M., Crielaard, W., Volgenant, C.M.C., van der Veen, M.H., Brandt, B.W. and Krom, B.P. (2017) Candida albicans alters the bacterial microbiome of early in vitro oral biofilms. J. Oral Microbiol., 9: 1270613.

7. Younis, G., Awad, A., Dawod, R.E. and Nehal, E. (2017) Antimicrobial activity of yeasts against some pathogenic bacteria. Vet. World, 10: 979-983.

8. Westerman, R.B., He, Y., Keen, J.E., Littledike, E.T. and Kwang, J. (1997) Production and characterization of monoclonal antibodies specific for the lipopolysaccharide of Escherichia coli O157. J. Clin. Microbiol., 35: 679-684.

9. Dumistrescu, A.L. (2006) Histological comparison of periodontal inflammatory changes in two models of experimental periodontitis the rat. A pilot study. Timisoara Med. J., 56: 211-217.

10. Li, S.D., Chen, Y.B., Qiu, L.G. and Qin, M.Q. (2017) G-CSF indirectly induces apoptosis of osteoblasts during hematopoietic stem cell mobilization. Clin. Transl. Sci., 10: 287-291.

11. Strålberg, F., Kassem, A., Kasprzykowski, F., Abrahamson, M., Grubb, A., Lindholm, C. and Lerner, U.H. (2017) Inhibition of lipopolysaccharide-induced osteoclast formation and bone resorption in vitro and in vivo by cysteine proteinase inhibitors. J. Leukoc. Biol., 101: 1233-1243.

12. Nirwana, I., Rachmadi, P. and Rianti, D. (2017) Potential of pomegranate fruit extract (Punica granatum Linn.) to increase vascular endothelial growth factor and platelet-derived growth factor expressions on the post-tooth extraction wound of Cavia Cobaya. Vet. World, 10: 999-1003.

13. Nair, S.P., Meghji, S., Wilson, M., Reddi, K., White, P. and Henderson, B. (1996) Bacterially induced bone destruction: Mechanisms and misconceptions. Infect. Immun., 64(7): 2371-2380. 
14. Schreiner, H., Markowitz, K., Miryalkar, M., Moore, D., Diehl, S. and Fine, D.H. (2010) Aggregatibacter actinomycetemcomitans-induced bone loss and antibody response in three rat strain. J. Periodontol., 82: 1-16.

15. Graves, D.T., Oates, T. and Garlet, G.P. (2011) Review of osteoimmunology and the host response in endodontic and periodontal lesion. J. Oral Microbiol., 3: 5304.

16. Nield-Gehrig, J.S. and Willmann, D.E. (2007) Foundation of Periodontics for the Dental Hygienist. Lippincott Williams \& Wilkins, Philadelphia, PA.
17. Claesson, R., Johansson, A., Belibasakis, G., Hänstrőm, L. and Kalfas, S. (2002) Release and activation of matrix metalloproteinase 8 from human neutrophils triggered by theleukotoxin of Actinobacillus actinomycetemcomitans. $J$. Periodontal. Res., 37: 353-359.

18. Bullon, P., Chandler, L., Segura Egea, J.J., Cano, R.P. and Sahuquillo, A.M. (2007) Osteocalcin in serum, saliva and gingival crevicular fluid: Their relation with periodontal treatment outcome in postmenopausal women. Med. Oral. Patol. Oral Cir. Bucal., 12(3): E193-E197.

\section{$* * * * * * * *$}

